\title{
QuMANET- changes in Mobile ad-hoc network with quantum bits for reliability
}

\author{
Shruti Mishra \\ Research Scholar \\ Bhagwant University, \\ Ajmer (Rajasthan), India
}

\author{
Dr. B. Dhanasekaran \\ Professor \\ SJIET \\ Chennai, India
}

\begin{abstract}
Due to the mobility, service discovery and service selection in Mobile Ad hoc Network (MANET), the routing protocol of MANET must adapt to the rapid changes of the network structure, and ensure that the services will be available to the users as quickly as possible. This paper proposes a kind of new quantum based MANET with the help of which service discovery will be fast enough that the user will not get a break in connection and swap over multiple services very fast with the help of quantum bits. By embedding MANET with quantum computing bits, it can effectively reduce the time for service selection, improve the delivery rate of data packets, and reduce the time delay of switching between services. The goal of this work is to shed light on the challenges and the open problems of the Quantum bits dealing with MANET design. To this aim, we first introduce some basic knowledge of Quantum mechanics, MANET needed to understand the differences between a MANET and the design we are thinking quantum MANET. Then, we introduce quantum bits as the key strategy for service swapping without physically transferring the particle that stores the quantum information. Finally, we will introduce the challenges that can be faced while transferring data over quantum bits.
\end{abstract}

Keywords: MANET, Quantum bits, mobility, service discovery, Qubits, QUMANET.

\section{INTRODUCTION}

Nowadays, Researchers are working with quantum computers and proposing new ideas about how successfully we can deal with quantum bits (qubits). On Jan 3,2020 Researchers at the Department of Energy's Oak Ridge National Laboratory simulated the performance of quantum devices. On May 18, 2020 university of California has set a new record for preparing and measuring the quantum bits, or qubits, inside of a quantum computer without error. The techniques they have developed make it easier to build quantum computers that outperform classical computers for important tasks [1]. Researchers are also working on quantum mechanical interactions. We can use networking in multiple quantum devices. Quantum MANET can be visualized to open a new way to provide fast services to the users.

All the researchers are in a race to work upon quantum computers and provide the best results in different fields. They are working on affordable quantum computers for real world business and government applications. [2] This goal achievement is crucial as to control interaction between different quantum systems in MANET remains extremely challenging.

We can create complementary approach towards mobile network to connect multiple clouds through wireless links. These small clouds will enable signals to interact with qubits even while supporting mobility.

Typically, MANET is built with no fixed infrastructure and routers. These are developed for temporary network connections. A mobile ad-hoc network (MANET) is a network supporting multi-hop with no fixed topology and offer different services to all the nodes. [3]
In this disquisition, we will focus on employment of qubits in MANET with the objective of fastest services that we can provide to the users.

The rest of the paper is structured as follows. In section 2 we will discuss the mechanics of quantum bits, their measurement, and quantum entanglement. We continue by offering a brief historical perspective of quantum computing and review the measurement of bits. In section 3, we will take a brief introduction of mobile ad-hoc network. In section 4 , we will describe the advancement in present mobile ad-hoc network with advent of qubits. Finally, we will do comparison in section 5 and study the various challenges in section 6 and then we will conclude in section 7 .

\section{QUANTUM MECHANICS}

Quantum computers are based on quantum mechanics and qubits increase the value of threshold at which information can be shared and processed. In classical computers, we were using binary bits 0 or 1 while in quantum computers we make use of both 0 and 1 by encoding them with in between values thus, providing large number of possibilities for storing data. As we know, with the help of 0 and 1 we can make only 4 combinations. But with the help of Qubits we can make $2^{\text {n }}$ combinations and we can do parallel processing practically with quantum computers.

Multiple quantum algorithms [4] are implemented to find a better solution to remove the various problems like delay in networks etc. However, dealing with superposition and entanglement is only one aspect of quantum computing. An equal challenge is to control the exponential bits in MANET which will be exciting time for breakthroughs in ad-hoc networks. This new technology can advance quickly if discovery of quantum internet will continue to push forward quantum technologies. 


\subsection{Quantum Entanglement}

It is a special case of superposition in which we define qubits as a reference to each other where the particles can be shared or they can be spatially separated. We can define physical properties of the system with the help of these qubits where one can influence other. For example, if two systems are connected to each other in a network and if we create influencing relationship between the two with the help of qubits where processing of one system influence other with the help of qubits then all the system will be connected with the help of quantum entanglement and MANET will work properly there.

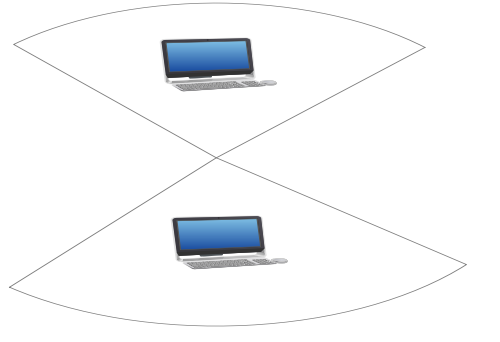

Figure 1 Quantum entanglement

Entanglement enables multiple applications since it helps to exchange of information very fast between qubits.

\subsection{Quantum measurement}

Quantum measurement can be considered as measuring the value of qubits that can be either 0 or 1 . We consider that qubits collapse towards either 0 or 1 . These outcomes are probabilistic. Superposition principle helps in determining the value of quantum states. This principle can be taken as a basis for quantum theory for determining states. We can add or superimpose multiple states to find another valid quantum state. This quantum state is a linear combination of multiple quantum states and these states can be either $|0\rangle$ or $|1\rangle$. These states are convertible with equal probability of $50 \%$ with $2^{\mathrm{n}}$ states.

\section{MANET}

Mobile ad-hoc network is a type of network that uses the concept of decentralization. There can be direct or indirect type of communication. When the nodes are in reach of one another, they can create direct communication while if they are located far from each other, they create communication through other nodes i.e indirect communication. There are multiple applications of MANET like military, health care etc. In MANET all the nodes are free to join and leave the network. All the nodes that are joining can take part in communication either as source or destination. Bandwidth is an important network property of wireless network because it is much lower in wireless link than that of wired links.

In this paper we are going to transform this MANET into QUMANET. This transformation is required as users of wireless network are increasing. As the pandemic COVID-19 has hit all the countries, uses of MANET have increased day by day. Users like students, teachers, company employees etc. all are accessing their facility from their home. So, it is the basic need of today that we should provide fast services to all the users

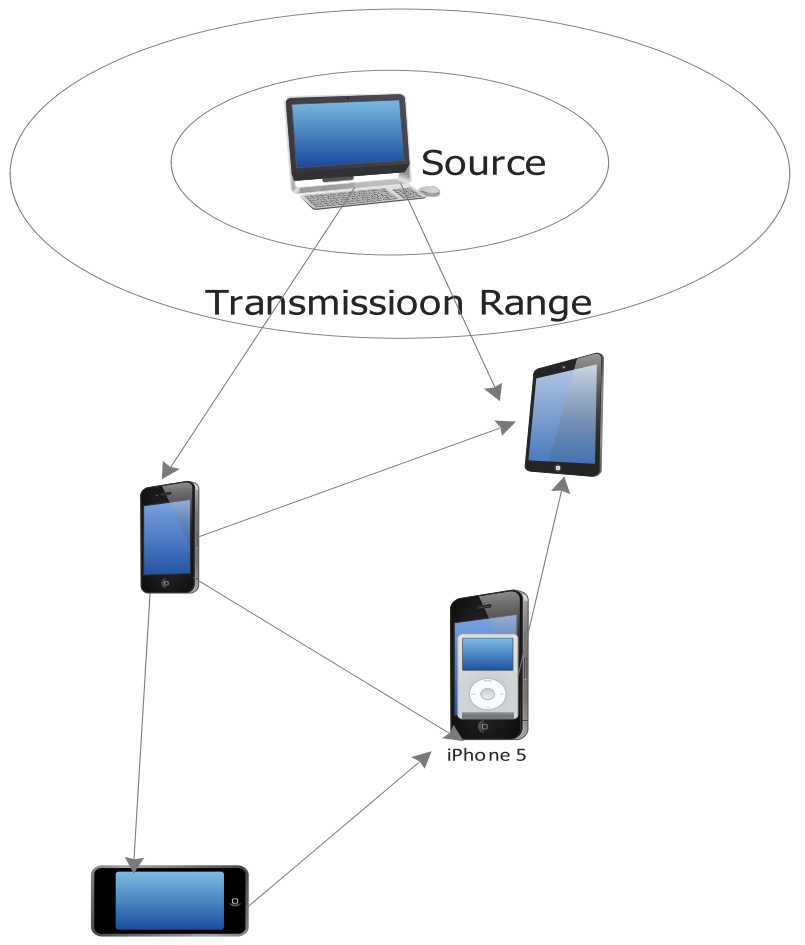

Figure 2: Mobile ad-hoc network

\section{OVERVIEW OF QUMANET}

In a classical MANET, the network provides QoS with the help of 0 and 1 . Whenever a node is moving from one point to another and wants to access some service with the help of service provider (SP), these services may be lost due to mobility. But with the advent of quantum adaptation, some applications like quantum key distribution (QKD) and superdense coding [5] are used. Quantum MANET can enhance the concept of quantum communication by using EPR pair also called bell states in superimposed state. If we try to measure these superimposed states qubits then we can find the equal distribution of 0or 1 with equal probability. This probability will help to distributed services in a network. Thus, we will be able to transfer messages with the help of these qubits. The EPR paradox used by Einstein and his colleagues helps to identify quantum entanglement behavior of qubits.

\section{COMPARISON}

There are different researches [8]-[10] that show Quantum internet can be used for long- distance communication of quantum and classical information. We can use this type of network in recent technology i.e MANET.

Services that are provided by different service providers and the number of services will be more in QUMANET as compared to classical MANET because the number of states will be increased in QUMANET. 


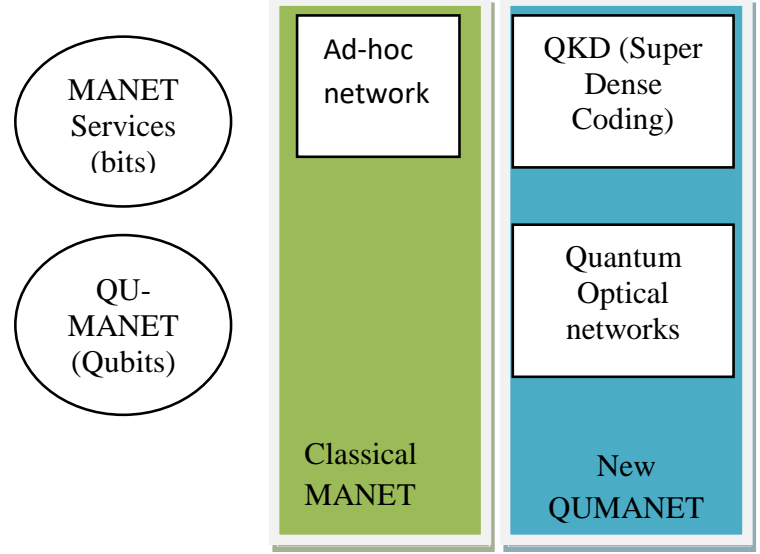

Figure 3: Classical MANET versus QUMANET

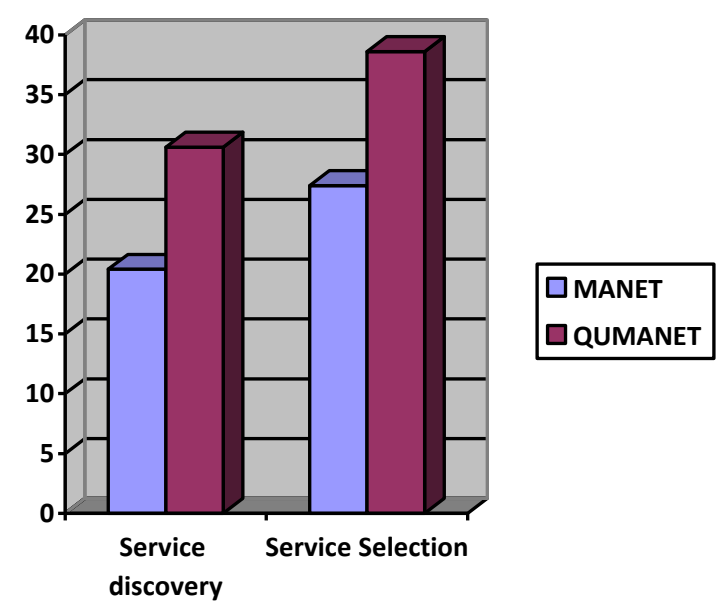

Figure 4: Comparison of MANET and QUMANET: Service discovery and selection with the help of QUMANET will be fast as compared to Classical MANET

\section{CHALLENGES}

Quantum MANET is still a complicated project to implement in real world as we have to find solutions of some challenges:

$\checkmark$ Implementation of QuMANET will require realization of qubits in small clusters for temporary time.

$\checkmark \quad$ Each time a node access the service with the help of quantum bits, it must be entangled to some states providing services to the users with security.

$\checkmark$ Some protocol must be implemented in ad-hoc network that must be followed for managing information required by different users.

\section{CONCLUSION}

QuMANET can be implemented by solving all these open problems. This is a very interesting concept including set of ideas that will help to improve speed, security, and facilities provided to the users. Quantum MANET will also help in implementation of advance networks like $6 \mathrm{G}$ and more.

\section{REFERENCES}

1. University of California - Los Angeles. "Physicists develop world's best quantum bits." ScienceDaily. ScienceDaily, 18 May 2020.

2. University of New South Wales. "Hot qubits break one of the biggest constraints to practical quantum computers." ScienceDaily. ScienceDaily, 15 April 2020.

3. Sharma, R.K.; Sharma, A.K.; Jain, V. Genetic Algorithm-Based Routing Protocol for Energy Efficient Routing in MANETs. In Next-Generation Networks; Lobiyal, D.K., Mansotra, V., Singh, U., Eds.; Springer: Singapore, 2018; Volume 638, pp. 33-40. ISBN 978-981-10-6004-5

4. Kindem, J.M., Ruskuc, A., Bartholomew, J.G. et al. Control and single-shot readout of an ion embedded in a nanophotonic cavity. Nature (2020).

5. M. A. Nielsen and I. L. Chuang, Quantum Computation and QuantumInformation, 10th ed. Cambridge University Press, 2011.

6. R. J. Lipton and K. W. Regan, Quantum Algorithms via Linear Algebra: A Primer. MIT Press, 2014.

7. Jozsa R, Linden N. On the role of entanglement in quantum computational speed-up. Proc Roy Soc Lond A, 2003, 459: 2011-2032

8. M. Caleffi, A. S. Cacciapuoti, and G. Bianchi, "Quantum internet: from communication to distributed computing!" in Proc. of IEEE/ACM ANOCOM, 2018, invited paper.

9. S. Pirandola and S. L. Braunstein, "Physics: Unite to build a quantum Internet," Nature, vol. 532, no. 7598, pp. 169-171, Apr. 2016.

10. S. Wehner, D. Elkouss, and R. Hanson, "Quantum internet: A vision for the road ahead," Science, vol. 362, no. 6412, Oct. 2018. 